\title{
Clinical outcomes of gastric polyps and neoplasms in patients with familial adenomatous polyposis
}

\section{(ㅇ)( $\odot$}

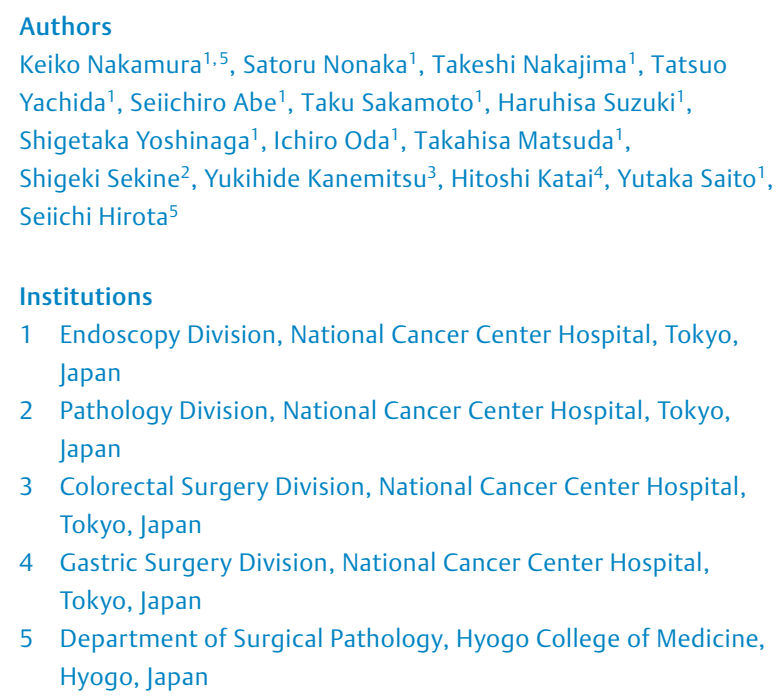

Keiko Nakamura1,5, Satoru Nonaka', Takeshi Nakajima'1, Tatsuo Yachida ${ }^{1}$, Seiichiro Abe ${ }^{1}$, Taku Sakamoto ${ }^{1}$, Haruhisa Suzuki ${ }^{1}$, Shigetaka Yoshinaga ${ }^{1}$, Ichiro Oda ${ }^{1}$, Takahisa Matsuda ${ }^{1}$, Shigeki Sekine ${ }^{2}$, Yukihide Kanemitsu ${ }^{3}$, Hitoshi Katai ${ }^{4}$, Yutaka Saito ${ }^{1}$, Seiichi Hirota ${ }^{5}$

Institutions

1 Endoscopy Division, National Cancer Center Hospital, Tokyo, Japan

2 Pathology Division, National Cancer Center Hospital, Tokyo, Japan

3 Colorectal Surgery Division, National Cancer Center Hospital, Tokyo, Japan

4 Gastric Surgery Division, National Cancer Center Hospital, Tokyo, Japan

5 Department of Surgical Pathology, Hyogo College of Medicine, Hyogo, Japan

submitted 25.5.2016

accepted after revision 4.10 .2016

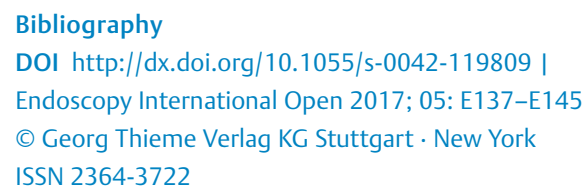

Corresponding author

Satoru Nonaka, Endoscopy Division, National Cancer Center

Hospital, 3.4.5F,8-1, Akashi-cho, 5-1-1 Tsukiji, Chuo-ku,

Tokyo 104-0045, Japan

Phone: +81-3-3542-2511

Fax: +81-3-3542-3815

snonaka@ncc.go.jp

\begin{abstract}
Background and study aims Familial adenomatous polyposis (FAP) is an autosomal dominant syndrome caused by a germline mutation in the adenomatous polyposis coli (APC) gene, characterized by the presence of more than 100 adenomatous polyps in the colorectum. The upper gastrointestinal tract is an extracolonic site for malignancy in patients with FAP. The frequency of death in Japanese patients with FAP because of gastric cancer is $2.8 \%$ and that because of colon cancer is $60.6 \%$. Few studies have reported upper gastrointestinal diseases in patients with FAP. In the present study, we investigated the clinical outcomes of patients with FAP diagnosed with gastric neoplasms.

Patients and methods We enrolled 80 patients with FAP who underwent esophagogastroduodenoscopy from October 1997 to December 2011. We investigated patient characteristics, endoscopic findings of gastric lesions, treatment outcomes, and long-term courses.

Results Fundic gland polyposis was observed in 51 patients (64\%) and gastric neoplasms in 22 patients ( $28 \%$ ), including 20 with noninvasive and 2 with invasive neoplasm. Of the 26 neoplasms, 11 were treated by endoscopic resection (ER) and 4 by surgical resection. Metachronous gastric neoplasms were observed in 7 patients (15 lesions) and treated by ER, except for in 1 patient. No patients died of gastric lesions during a median follow-up period of 6.5 years (range, $0-14$ ).

Conclusion Because gastric lesions including gastric cancers in patients with FAP did not cause any deaths, they can be considered to have favorable prognoses. Early detection of gastric neoplasms through an appropriate follow-up interval may have contributed to these good outcomes.
\end{abstract}

\section{Introduction}

Familial adenomatous polyposis (FAP) is an autosomal dominant syndrome caused by a mutation in the adenomatous polyposis coli (APC) gene characterized by the presence of more than 100 adenomatous polyps in the colorectum. The penetrance of colorectal cancer in patients with FAP in their $40 \mathrm{~s}$ is approximately $50 \%$ and rises to approximately $100 \%$ if they are not treated before their $60 \mathrm{~s}$ [1]. With the exception of colorectal cancer, various neoplastic and non-neoplastic lesions occur in other organs in patients with FAP. The most frequent cause of death in patients with FAP is colorectal cancer, which accounts for $60.6 \%$ of FAP-related deaths in Japan [2]. As the prognoses of patients with FAP have improved in recent years because of the use of prophylactic colectomy, postoperative extracolonic lesions have started to become a more significant issue.

The causes of death in Japanese patients with FAP because of lesions outside the colon are as follows: desmoid neoplasms in $9.9 \%$, duodenal cancer in $5.6 \%$, and gastric cancer in $2.8 \%$ cases [2]. Since the first report of complicating gastric lesions in patients with FAP by Hauser in 1895 [3], a high proportion of synchronous neoplasms have been reported [2,4-6]. The types of gastric lesions associated with FAP are fundic gland polyps, gastric adenomas, and gastric cancers. Fundic gland polyposis 
- Table 1 Characteristics of patients with FAP.

\begin{tabular}{|c|c|}
\hline Patient, no. & 80 \\
\hline Age at initial EGD (mean \pm SD, years) & $40 \pm 17$ \\
\hline Male, no. (\%) & $52(65)$ \\
\hline Female & $28(35)$ \\
\hline Present of familial history of FAP, no. (\%) & $42(53)$ \\
\hline \multicolumn{2}{|l|}{ Types of FAP, no. (\%) } \\
\hline Classical & $69(86)$ \\
\hline Profused & $11(14)$ \\
\hline \multicolumn{2}{|l|}{ Treatment, no. (\%) } \\
\hline Total colectomy & $68(85)$ \\
\hline Other & $3(4)$ \\
\hline Observation without surgical procedure & $9(11)$ \\
\hline Follow-up period, years, median (range) & $6.5(0-14)$ \\
\hline \multicolumn{2}{|l|}{ Follow-up interval by EGD, months } \\
\hline 12 & $45(56)$ \\
\hline 18 & $13(16)$ \\
\hline 24 & $7(9)$ \\
\hline None & $12(15)$ \\
\hline Other $^{1}$ & $3(4)$ \\
\hline \multicolumn{2}{|l|}{ Fundic gland polyps, no. $(\%)^{2}$} \\
\hline Present & $51(64)$ \\
\hline$<100$ & $12(15)$ \\
\hline$\geq 100$ & $39(49)$ \\
\hline Absent & $29(36)$ \\
\hline Gastric neoplasm, no. $(\%)^{3}$ & $22(28)$ \\
\hline \multicolumn{2}{|c|}{$\begin{array}{l}\text { FAP, familial adenomatous polyposis; EGD, esophagogastroduodenoscopy } \\
{ }^{1} \text { The follow-up interval for } 3 \text { patients was } 6 \text { months, } 36 \text { months, and } 42 \\
\text { months, respectively } \\
{ }^{2} \text { Some patients had both fundic gland polyps and gastric neoplasms. } \\
{ }^{3} \text { Gastric neoplasm included invasive neoplasm and noninvasive neoplasm }\end{array}$} \\
\hline
\end{tabular}

(FGP) associated with FAP was first reported by Halstead et al. and is the most common gastric lesion in patients with FAP, showing an incidence of 50\%-60\% [7-9]. Gastric adenoma in patients with FAP was initially reported by Hoffman et al. [10], and several studies have reported the incidence of gastric adenoma as $2 \%-35 \%$ [7, $11-13]$. Gastric cancer related to FAP was first reported by Murphy et al. [14]. In Japan, gastric cancer is observed in $4.5 \%-13.6 \%$ patients with FAP [15], whereas the incidence in East Asia is $2.7 \%-4.2 \%[13,16]$. In western countries, the incidence of gastric cancer associated with FAP is similar to that of the general population [17], whereas that in East Asia is $3-4$ times higher $[15,16]$. However, few reports about the clinical course and treatment of gastric lesions in patients with FAP exist. Therefore, we aimed to investigate the clinical outcomes of patients with FAP with gastric lesions and to reveal the relationships between the incidence of gastric neoplasms and the background mucosa.

\section{Patients and methods}

We retrospectively investigated 80 patients with FAP who underwent esophagogastroduodenoscopy (EGD) as a regular surveillance examination in 142 patients diagnosed with FAP from October 1997 to December 2011 at the National Cancer Centre Hospital, Tokyo, Japan. The institutional review board of the National Cancer Centre Hospital approved the study, and we obtained informed consent from all patients. We investigated patient characteristics, endoscopic findings of gastric lesions, treatment outcomes, and clinical courses, and information regarding all gastric lesions was described in accordance with the Japanese Classification of Gastric Carcinoma (JCGC) [18]. Gastric neoplasms detected over 1 year after initial endoscopic resection (ER) or surgery were defined as metachronous gastric neoplasms (MGNs). The endoscopic extent of gastric atrophy was diagnosed according to the Kimura-Takemoto classification, which correlates with the histologic degree of atrophic gastritis [19]. Patients with FAP having atrophic gastritis were regarded as having a history of Helicobacter pylori infection. The condition of the background gastric mucosa was classified into 4 groups according to the presence of FGP and atrophic gastritis as follows: FGP with atrophic gastritis, FGP without atrophic gastritis, no FGP with atrophic gastritis, and no FGP without atrophic gastritis. Gastric neoplasms included invasive neoplasms and noninvasive neoplasms; invasive neoplasms were defined as gastric cancers invading the submucosal layer or deeper or poorly differentiated adenocarcinoma, and noninvasive neoplasms were defined as gastric adenomas or intramucosal carcinomas. Two expert pathologists reviewed all resected specimens at the National Cancer Center Hospital. Histologic diagnoses were based on the Japanese Classification of Gastric Carcinoma [18].

Treatment strategies for gastric cancers were determined by a cancer board consisting of endoscopists, surgeons, and pathologists. Patients with gastric cancer were treated based on the Japanese Gastric Cancer Treatment Guidelines [20]. The basic hospital policy for ER for gastric neoplasm is to resect all lesions containing biopsy-confirmed adenocarcinoma. Therefore, all patients with FAP having gastric adenoma underwent regular surveillance EGD and provided biopsy specimens from gastric adenomas regardless of macroscopic changes. ER included endoscopic submucosal dissection (ESD) and endoscopic mucosal resection (EMR) [21-24]. All ER procedures and surgeries were performed by skilled endoscopists and surgeons at the National Cancer Center Hospital.

Baseline and outcomes data for patients who underwent ER were collected from electronic medical records and prospectively collated in a computerized database. In addition, we analyzed the proportion of patients with FAP who underwent surgical colectomy between 1972 and 2010 in cooperation with the colorectal surgery division. Pathologic information was subsequently added after ER or surgical resection results were confirmed. All patients with FAP were followed up every 6 months 
- Table 2 Characteristics and clinical outcomes of primary gastric neoplasms (22 patients with 26 lesions ${ }^{1}$ ).

\begin{tabular}{|c|c|}
\hline Patient, no. & 22 \\
\hline $\begin{array}{l}\text { Age at the time of diagnosis with gastric neoplasm, } \\
\text { years, mean } \pm \text { SD }\end{array}$ & $46 \pm 12$ \\
\hline Family history of FAP, no. (\%) & $7(32)$ \\
\hline $\begin{array}{l}\text { Period from prophylactic colectomy to occurrence of } \\
\text { gastric neoplasm, years, median (range) }\end{array}$ & $18(2-35)$ \\
\hline \multicolumn{2}{|l|}{ Findings of background gastric mucosa, no. (\%) } \\
\hline FGP with atrophic gastritis & $2(9)$ \\
\hline FGP without atrophic gastritis & $8(36)$ \\
\hline Non-FGP with atrophic gastritis & $11(50)$ \\
\hline Non-FGP without atrophic gastritis & $1(5)$ \\
\hline \multicolumn{2}{|l|}{ Total number of gastric neoplasms², no. (\%) } \\
\hline 1 & $15(68)$ \\
\hline 2 & $4(18)$ \\
\hline 3 & $1(5)$ \\
\hline$\geq$ & $2(9)$ \\
\hline \multicolumn{2}{|l|}{ Location, no. (\%) ${ }^{1}$} \\
\hline Upper two-thirds & $19(73)$ \\
\hline Lower one-third & $7(27)$ \\
\hline \multicolumn{2}{|l|}{ Macroscopic type, no. $(\%)^{1}$} \\
\hline Elevated & $17(65)$ \\
\hline Depressed & $7(27)$ \\
\hline Combined & $1(4)$ \\
\hline Advanced $^{3}$ & $1(4)$ \\
\hline Neoplasm size, mm, median (range) & $10(3-70)$ \\
\hline \multicolumn{2}{|l|}{ Treatment, no. $(\%)^{1}$} \\
\hline Endoscopic resection & $11(42)$ \\
\hline EMR & $7(27)$ \\
\hline ESD & $4(15)$ \\
\hline Surgery & $4(15)$ \\
\hline Observation & $12(46)$ \\
\hline \multicolumn{2}{|l|}{ Histologic type, no. $(\%)^{1}$} \\
\hline Adenoma & $12(46)$ \\
\hline Differentiated-type adenocarcinoma (tub1/tub2) & $14(54)$ \\
\hline \multicolumn{2}{|l|}{ Neoplasm depth, no. $(\%)^{1}$} \\
\hline Intramucosal & $24(92)$ \\
\hline Submucosa or deeper & $2(8)$ \\
\hline \multicolumn{2}{|c|}{$\begin{array}{l}\text { FAP, familial adenomatous polyposis; FGP, fundic gland polyposis; EMR, } \\
\text { endoscopic mucosal resection; ESD, endoscopic submucosal dissection; } \\
\text { tub1, well-differentiated tubular adenocarcinoma; tub2, moderately differ- } \\
\text { entiated tubular adenocarcinoma. } \\
{ }^{1} \text { Two patients who had multiple gastric adenomas were excluded because } \\
\text { the large number of lesions were difficult to count exactly. } \\
{ }^{2} \text { Total number of gastric neoplasms excluded metachronous neoplasms. } \\
{ }^{3} \text { Type } 2 \text { in Japanese Gastric Cancer Treatment Guidelines. }\end{array}$} \\
\hline
\end{tabular}

or 12 months with blood tests, EGD, and computed tomography.

\section{Results}

\section{Characteristics of patients with FAP}

The mean age of the 80 patients ( 52 men and 28 women) at initial EGD was $40 \pm 17$ years (mean \pm SD) ( $\triangleright$ Table 1 ). Of them, 42 (53\%) had an obvious family history of FAP. The median followup period was 6.5 years (range, $0-14$ ). Surveillance EGD was generally administered at intervals of 12 months at the National Cancer Center Hospital, and 45 patients (56\%) underwent EGD at this interval.

\section{Patient characteristics, endoscopic findings, and clinical course}

FGP was observed in 51 patients (64\%) and gastric neoplasms in $22(28 \%)$ ( Table 1), including noninvasive neoplasms in 20 patients and invasive neoplasms in 2.

In total, 26 gastric neoplasms in 22 patients were detected during the follow-up period, excluding MGNs ( $\triangleright$ Table 2 ). The mean age at diagnosis with gastric neoplasms was $46 \pm 12$ years (mean \pm SD), and 7 patients (32\%) had a family history of FAP. The median period from colectomy to the development of gastric neoplasms was 18 years (range, 2-35). Regarding the background gastric mucosa in the cases in which gastric neoplasms were detected, 11 cases (50\%) occurred in patients with no FGP with atrophic gastritis, and 8 cases (36\%) in those with FGP without atrophic gastritis ( $>$ Fig.1). The number of primary gastric neoplasms was 1 in 15 patients (68\%), 2 in 4 patients (18\%), 3 in 1 patient (5\%), and 4 or more in 2 patients (9\%). The location was the upper two-thirds of the stomach for 19 lesions (73\%). The macroscopic type was elevated for 17 lesions (65\%), depressed for 7 lesions (27\%), and advanced (Type 2 in JGCA) for 1 lesion (4\%). The median neoplasm size was $10 \mathrm{~mm}$ (range, $3-70$ ) and the histopathologicl findings revealed adenoma in 12 lesions (46\%) and differentiated-type adenocarcinoma in 14 lesions (54\%). The depth of gastric neoplasm invasion was intramucosal in 24 lesions (92\%) and the submucosa or deeper in two lesions ( $8 \%$ ).

We illustrated 2 cases of gastric neoplasms, the first case had a fundic gland polyp developing gastric neoplasm ( $\triangleright$ Fig.2); and the second case had an intramucosal gastric neoplasm in the background gastric mucosa of FGP ( $\triangleright$ Fig. 3 ). The first case was a male patient who was diagnosed with FAP at age 31 and underwent subtotal colectomy for rectal cancer. His mother also had FAP. EGD revealed a fundic gland polyp on the lesser curvature of the middle gastric body ( $\vee$ Fig. 2a). Surveillance EGD was conducted at 18-month intervals. By age 35, lesion size had increased ( $>$ Fig. $\mathbf{2 b}$ ). By age 38 , the lesion had grown further, turned red, and developed an erosive change in its center ( $>$ Fig. $\mathbf{2 c}$ ). Biopsy revealed a well-differentiated adenocarcinoma (tub1) with low-grade atypia, and ESD was performed for the elevated lesion and resulted in successful en bloc resection. Pathological result revealed Type 0-Ila, $12 \mathrm{~mm}$, well-differentiated adenocarcinoma, pT1a (M), ly(-), v(-), pHM0, pVM0 ( $\triangleright$ Fig. 2d). The second case was a female patient who was di- 


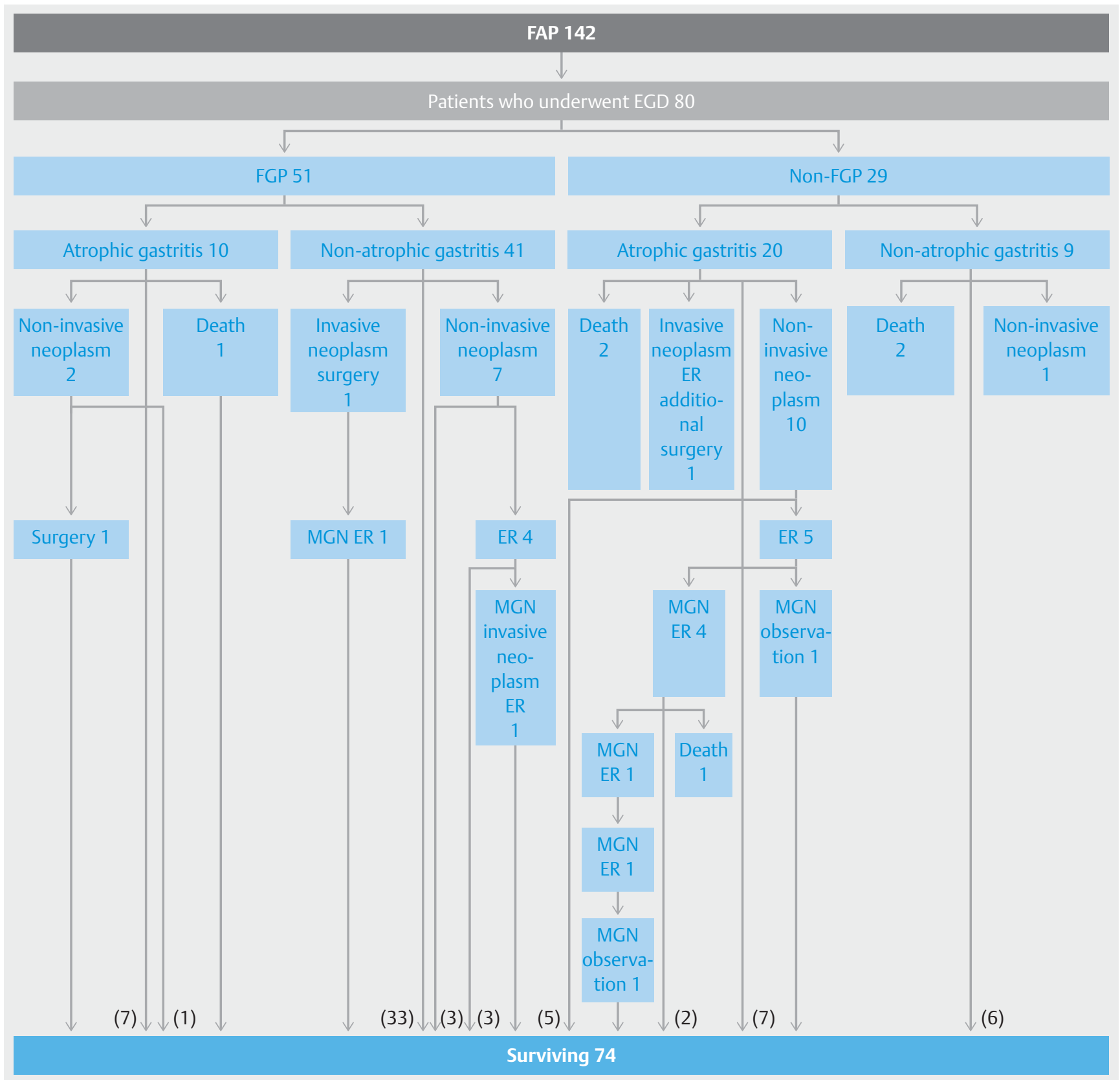

Fig. 1 Flow diagram of the clinical courses of 80 patients with FAP who underwent EGD. The clinical courses were classified according to the background gastric mucosa. Invasive neoplasms were defined as gastric cancers invading deeper than the submucosal layer and poorly differentiated adenocarcinoma, and noninvasive neoplasms were defined as gastric adenomas and intramucosal carcinomas. FAP, familial adenomatous polyposis; EGD, esophagogastroduodenoscopy; FGP, fundic gland polyposis; ER, endoscopic resection; MGN, metachronous gastric neoplasm; $\mathrm{Cl}$, cerebral infarction.

agnosed with FAP and underwent prophylactic colectomy at age 20 . Her father and grandfather also had FAP. She received annual EGD beginning at age 20. At age 26, a whitish elevated lesion was identified on the anterior wall of the middle gastric body ( $\vee$ Fig.3a and $\vee$ Fig.3b). The biopsy revealed a tubular adenoma, but malignancy was suspected on the basis of the macroscopic appearance of the coalescent tendency of several polyps and its whitish color. The pathologic result of EMR was as follows: Type 0 -Ila, $10 \mathrm{~mm}$, well-differentiated adenocarcinoma, pT1a (M), ly(-), v(-), pHM0, pVM0 ( Fig.3c). At age 32, a tiny depressed signet-ring cell carcinoma (sig) without atrophic gastritis was detected in the posterior wall of the gastric antrum, and ESD was performed ( $\triangleright$ Fig. $\mathbf{3 d}$ ). Pathologic result at that time was as follows: Type $0-11$ c, $3 \mathrm{~mm}$, sig, pT1a (M), ly(-), v(-), pHM0, pVM0 ( $>$ Fig. 3e).

\section{Treatment outcomes of gastric neoplasms}

Of the 26 neoplasms, 14 primary gastric neoplasms (54\%) were treated ( $\triangleright$ Table 2 ). ER was performed for 11 lesions (42\%), and surgery was conducted for 4 lesions (15\%) although they were 


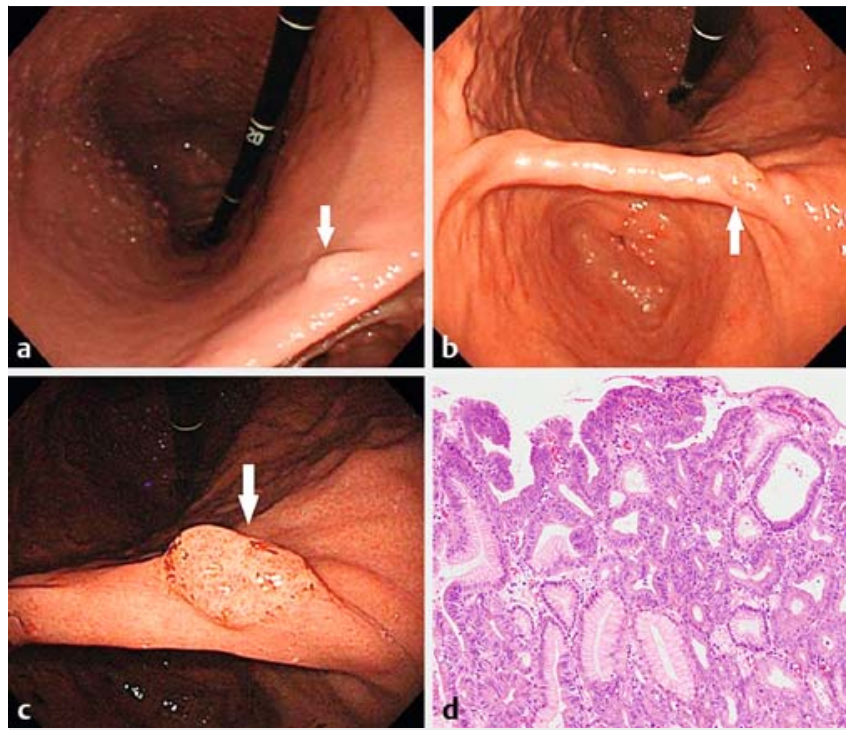

- Fig. 2 a Endoscopic findings of a male patient diagnosed as FAP at the age of 31 years. EGD revealed a fundic gland polyp on the lesser curvature of the middle gastric body. $\mathbf{b}$ By age 35 , lesion size had increased. $c$ By the age of 38 , the lesion had grown further, turned red, and developed an erosive change in its center. $\mathbf{d}$ Pathologic result: Type 0 -lla, $12 \mathrm{~mm}$, tub1 (foveolar adenoma in WHO histological classification), pT1a (M), ly(-), v(-), pHM0, pVM0. tub1, well-differentiated adenocarcinoma; $\mathrm{PT1a}(\mathrm{M})$, intramucosal cancer; HM, horizontal margin; VM, vertical margin; WHO, World Health Organization.

followed up regularly. In the 3 patients with 4 lesions who underwent surgery, the first patient had an invasive neoplasm in the upper body (0-Ilc, $10 \mathrm{~mm}$ ), which was diagnosed as a noninvasive neoplasm before ER and treated by ESD, resulting in noncurative resection by histopathology with submucosal invasion $(400 \mu \mathrm{m})$ and lymphovascular invasion. It was then necessary to perform additional surgery. In the second patient, 2 noninvasive neoplasms were observed in the upper body $(0-I I a+I l c$, $70 \mathrm{~mm}$, T1a [M]; 0-Ila, 44 mm, T1a [M]) and treated by proximal gastrectomy because of technical difficulties in performing ER in the fornix. The third patient had an invasive gastric neoplasm in the upper body (Type 2, $58 \mathrm{~mm}$ ) with liver metastases treated by partial gastrectomy and simultaneous left lateral liver segmentectomy. After 7 years, MGN (0-Ila, $2 \mathrm{~mm}$ ) was detected and treated by ER.

\section{Long-term course of gastric neoplasms}

Fifteen MGNs were detected in 7 patients, including 2 cases (29\%) in FGP without atrophic gastritis and 5 (71\%) in nonFGP with atrophic gastritis ( $\triangleright$ Table 3 ). The macroscopic types of MGNs were elevated for 7 lesions (47\%) and depressed for 7 lesions (47\%). The median period from primary gastric neoplasm to MGN was 4 years (range, $2-12$ ) and the median size of MGNs was $10 \mathrm{~mm}$ (range, 2-82). The site of MGNs was the upper two-thirds of the stomach in 7 lesions (47\%), and the lower third in 8 lesions (53\%). Ten of 15 MGNs were detected as noninvasive neoplasms (one adenoma and nine differentiated-type adenocarcinomas) and treated by ER. One MGN was detected as an invasive neoplasm because of histologic findings of an undifferentiated carcinoma $3 \mathrm{~mm}$ in size, but was successfully treated by ER. In contrast, 4 MGNs were observed because of histologic findings of adenoma for 3 lesions and at the patient's request for 1 differentiated-type adenocarcinoma.

In the long-term follow-up period, 3 patients died of colon cancer, 1 because of a desmoid neoplasm and 2 because of other diseases. Three patients who underwent surgical colectomy in the past died of metachronous colon cancer or liver metastasis of colon cancer: 1 patient in the remnant rectum 10 years later, 1 in the liver 6 months later, and 1 in the remnant rectum and the liver 23 years later. No deaths caused by gastric cancer occurred during the study period.

\section{Discussion}

The current study included the largest number of gastric lesions in patients with FAP and the longest follow-up period, considering all studies of upper gastrointestinal diseases in patients with FAP to date. The main finding was that no deaths attributable to gastric neoplasms occurred, suggesting that early detection based on an appropriate examination interval results in excellent prognoses.

In this study, gastric neoplasms occurred in $28 \%$ of patients (22/80) with FAP, with a total of 41 lesions including 15 MGNs, indicating that it is a complication with a high incidence rate. The features of primary gastric neoplasms associated with FAP included the macroscopic type of elevated lesions (65\%), location in the upper two-thirds of the stomach (73\%), the presence of 1 or 2 lesions (86\%), and histologic findings of differentiated-type adenocarcinoma (54\%) or adenoma (46\%). As for the location of gastric neoplasms, most of the general gastric cancers occur in the lower two-thirds of the stomach related to Helicobacter pylori infection. However, poorly differentiated adenocarcinomas develop more in the upper gastric body than the lower two-thirds of the stomach. They also occur in the border between the normal mucosa and atrophic mucosa associated with $H$. pylori infection. In general, the incidence of gastric cancers in the upper stomach was approximately $20 \%$; therefore, it is possible to explain the higher incidence of neoplastic lesion (73\%) in the proximal stomach in our study. Furthermore, eleven of 22 patients with FAP (50\%) had complicated gastric neoplasms in non-FGP with atrophic gastritis. However, eight of 22 patients (36\%) had gastric neoplasms in FGP without atrophic gastritis. Therefore, not only $H$. pylori but also FGP may be related to the occurrence of gastric neoplasms in patients with FAP. And high rate of well differentiated adenocarcinoma in gastric cancers of patients with FAP was consistent with previous reports $[25,26]$.

FGP was observed in $64 \%(51 / 80)$ of FAP cases and was seen in the gastric body and fundus. FGP has traditionally been recognized as hamartomatous lesions with no malignant potential that arise from the fundic gland region. However, invasive neoplasms occurred in two patients (5\%) and noninvasive neoplasms in 8 patients (20\%) in FGP without atrophic gastritis, suggesting that FGP has malignant potential. Several reports 

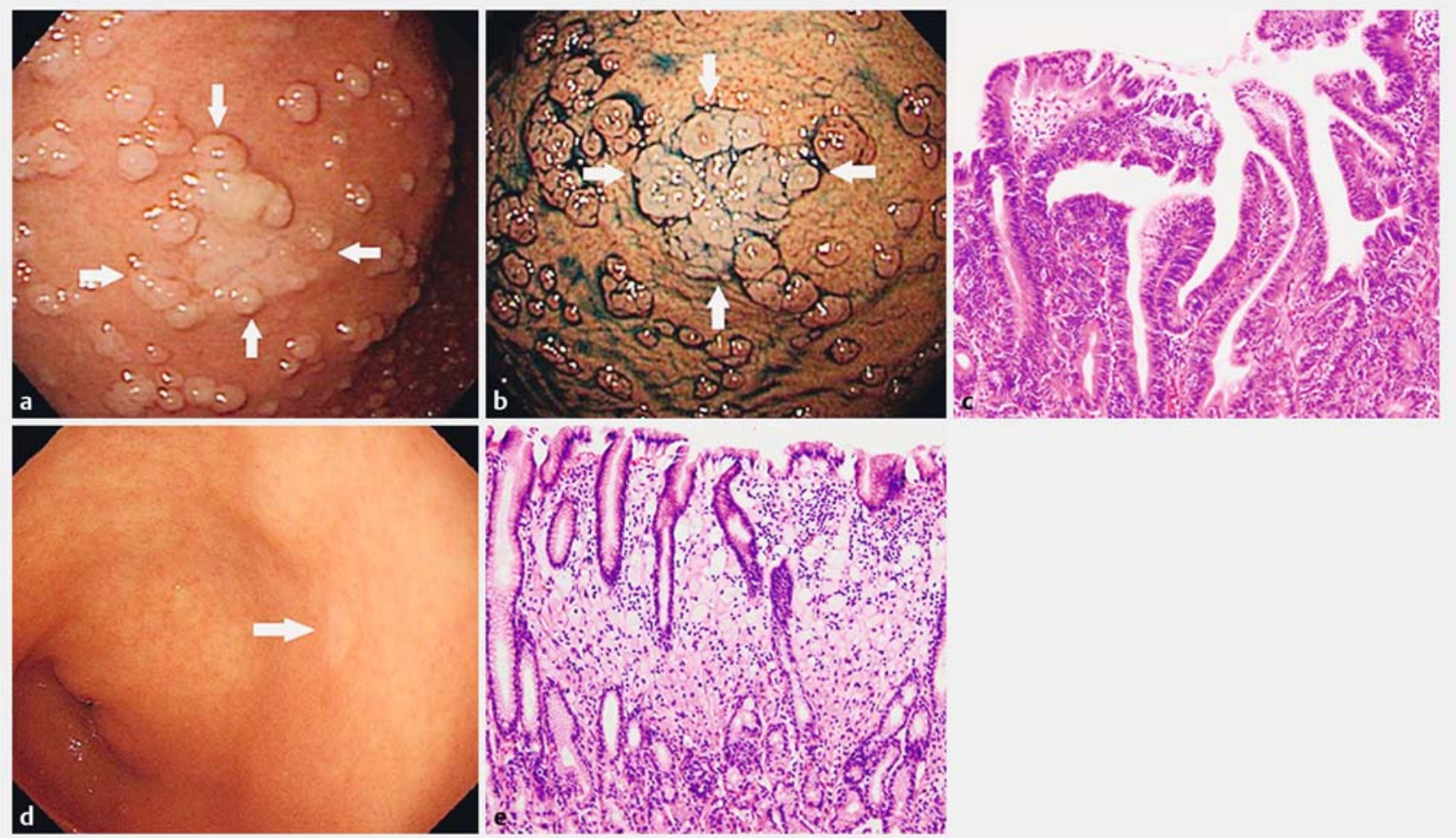

Fig. 3 Endoscopic findings of a female patient diagnosed with FAP at the age of 20 years. a, b At 26 years, a whitish elevated lesion was identified on the anterior wall of the middle gastric body in the background mucosa of FGP. c The pathological result of EMR was as follows: Type 0-Ila, $10 \mathrm{~mm}$, tub1 (foveolar adenoma in WHO histological classification), pT1a (M), ly(-), v(-), pHM0, pVM0. d At 32 years, a tiny depressed signet-ring cell carcinoma (sig) was detected in the posterior wall of the gastric antrum. e Pathologic result was as follows: Type 0-Ilc, $3 \mathrm{~mm}$, sig (signet-ring cell carcinoma in WHO histological classification), pT1a (M), ly(-), v(-), pHM0, pVM0. tub1, well-differentiated adenocarcinoma; PT1a (M), intramucosal cancer; HM, horizontal margin; VM, vertical margin; WHO, World Health Organization.

have described dysplasia in the foveolar epithelium of FAP-associated FGP $[27,28]$. Furthermore, some case reports have described high-grade dysplasia or adenocarcinoma in association with FAP-associated FGP, suggesting that FAP-associated FGP is a potential premalignant lesion [29-31]. As for genetic analysis, previous studies revealed the presence of somatic APC mutations in FAP-associated FGP, indicating neoplastic lesions caused by the inactivation of both alleles, similar to the mechanism of other FAP-associated neoplasms [28,32]. Consistent with this, 1 patient with 2 noninvasive neoplasms in FGP who underwent proximal gastrectomy in our study had inactivation of the APC gene as previously reported [31]. The macroscopic characteristics of FGP that developed into gastric neoplasms include a tendency for the coalescence of individual polyps, discoloration, and flattening. In addition, 9 of 10 patients with FAP (90\%) having gastric neoplasms in the background gastric mucosa with FGP had more than 100 fundic gland polyps. For the treatment, it was impractical to resect all fundic gland polyps because the number was numerous; 49\% (39/51) patients with FGP had more than 100 fundic gland polyps. Therefore, we detected the fundic gland polyps that showed a tendency for coalescence of individual polyp, discoloration, and flattening, suggesting the development of neoplastic lesions, and performed endoscopic resection for the lesions diagnosed as adenocarcinoma by biopsy. In addition, we treated the gastric neoplasms in the background of polyposis based on the Japanese Gastric Cancer Treatment Guidelines. Similar to general gastric cancers, intramucosal gastric neoplasms were resected by endoscopic resection (EMR/ESD) and invasive gastric neoplasms were resected by surgery. However, we performed surgical resection of the large noninvasive gastric neoplasms in the fornix or greater curvature of the upper gastric body after consulting surgeon because of the technical difficulty.

Mean age at the time of diagnosis of gastric neoplasms in patients with FAP in this study was $46 \pm 12$ years, which was younger than that in the general population [33]. We recommend EGD screening to be initiated at approximately age 25, because patients with FAP had gastric adenoma starting as early as age 24 and gastric cancer as early as age 25 in our study. EGD screening should be continued even after surgical colectomy in patients with FAP, as the median period from surgical colectomy to the occurrence of gastric neoplasms was 18 years (range, 2-35). According to the previous report, one of the reasons for the high incidence of gastric neoplasms in our study is related to the long-term follow-up [25]. As for the interval of surveillance EGD, most patients with $\operatorname{FAP}(73 \%, 58 / 80)$, including those with gastric neoplasms, underwent a procedure every 12 or 18 months in this study. We consider this interval to be 
- Table 3 haracteristics and clinical outcomes of metachronous gastric neoplasms (seven patients with 15 lesions).

\begin{tabular}{|c|c|}
\hline Patient, no. & 7 \\
\hline Present of family history of FAP, no. (\%) & $2(29)$ \\
\hline $\begin{array}{l}\text { Period from primary gastric neoplasm to occurrence } \\
\text { of metachronous neoplasms, years, median (range) }\end{array}$ & $4(2-12)$ \\
\hline \multicolumn{2}{|l|}{ Findings of background gastric mucosa, no. (\%) } \\
\hline FGP with atrophic gastritis & $0(0)$ \\
\hline FGP without atrophic gastritis & $2(29)$ \\
\hline Non-FGP with atrophic gastritis & $5(71)$ \\
\hline Non-FGP without atrophic gastritis & $0(0)$ \\
\hline \multicolumn{2}{|l|}{$\begin{array}{l}\text { Total number of metachronous gastric neoplasms, } \\
\text { no. (\%) }\end{array}$} \\
\hline 1 & $4(57)$ \\
\hline 2 & $1(14)$ \\
\hline $3-5$ & $1(14)$ \\
\hline 6 & $1(14)$ \\
\hline \multicolumn{2}{|l|}{ Location, no. (\%) } \\
\hline Upper two-thirds & $7(47)$ \\
\hline Lower one-third & $8(53)$ \\
\hline \multicolumn{2}{|l|}{ Macroscopic type, no. (\%) } \\
\hline Elevated & $7(47)$ \\
\hline Depressed & $7(47)$ \\
\hline Combined & $1(7)$ \\
\hline Advanced & $0(0)$ \\
\hline Neoplasm size¹, mm, median(range) & $10(2-82)$ \\
\hline \multicolumn{2}{|l|}{ Treatment, no. (\%) } \\
\hline Endoscopic resection & $11(73)$ \\
\hline EMR & $2(13)$ \\
\hline ESD & $9(6)$ \\
\hline Surgery & $0(0)$ \\
\hline Observation ${ }^{2}$ & $4(27)$ \\
\hline \multicolumn{2}{|l|}{ Histologic type, no. (\%) } \\
\hline Adenoma & $4(27)$ \\
\hline Differentiated-type adenocarcinoma (tub1/tub2) & $10(67)$ \\
\hline Undifferentiated adenocarcinoma (por/sig) & $1(7)$ \\
\hline \multicolumn{2}{|l|}{ Neoplasm depth, no. (\%) } \\
\hline Intramucosal & $15(100)$ \\
\hline Submucosa or deeper & $0(0)$ \\
\hline
\end{tabular}

FAP, familial adenomatous polyposis; FGP, fundic gland polyposis; EMR, endoscopic mucosal resection; ESD, endoscopic submucosal dissection; tub1, well-differentiated tubular adenocarcinoma; tub2, moderately differentiated tubular adenocarcinoma; por, poorly differentiated adenocarcinoma; sig, signet-ring cell carcinoma.

${ }^{1}$ The largest size in multiple lesions

24 metachronous gastric neoplasms were observed because of the histology of adenoma for 3 lesions and the patients request for one differentiatedtype adenocarcinoma. appropriate because none of the patients with FAP died of gastric neoplasms, and 38 of 41 (93\%) gastric neoplasms including MGNs were detected at the non-invasive stage. In terms of the background gastric mucosa, we carefully observe patients with FAP in the background mucosa of FGP and/or atrophic gastritis, in which incidence of gastric neoplasms was 95\% (21/22 patients). On the other hand, it is difficult to decide the appropriate interval for EGD screening for patients without FGP and atrophic gastritis in the background gastric mucosa because there was only 1 patient who had gastric neoplasms in background gastric mucosa without FGP and atrophic gastritis in our study. However, they should follow-up duodenal lesions by EGD screening annually because of the high risk of duodenal neoplasia [34]. Therefore, even gastric lesions can be followed up in the same session. In addition, the major duodenal papilla is also at risk for adenomas in patients with FAP. We reported the risk of duodenal neoplasia $(61 \%, 47 / 77)$ including ampullary neoplasms $(22 \%, 17 / 77)$, and we recommended a shortterm EGD surveillance for duodenal neoplasms including ampullary neoplasms [34].

Patients with FAP who have primary gastric neoplasms tend to have MGNs (32\%, 7/22 patients); the rate of MGNs is higher than that in previous reports $[35,36]$. With respect to the background gastric mucosa, MGNs were correlated with atrophic gastritis more than FGPs, demonstrated by the finding that 5 patients (71\%) had MGNs in non-FGP with atrophic gastritis, whereas only 2 patients $(29 \%)$ had MGNs in FGP without atrophic gastritis. The features of MGNs were as follows: located in the whole stomach, of the depressed macroscopic type, and elevated. As for the clinical course of MGNs, it was possible to control MGNs if regular surveillance EGD was performed after treatment for primary gastric neoplasms because most of them $(93 \%, 14 / 15)$ were detected at the noninvasive stage, excluding 1 undifferentiated adenocarcinoma.

Unfortunately, 6 of 80 patients with FAP died: colorectal cancer in 3 instances, a desmoid neoplasm in 1, cerebral infarction in 1, and an unknown cause in 1 . However, the remaining patients are still alive, and their life expectancy is longer than it would have been without surgical colectomy.

In the future, the risk of neoplasms other than colorectal cancer may increase. Therefore, all neoplasms associated with FAP including gastric neoplasms should be carefully observed. However, in this study, 24 of 26 gastric neoplasms (92\%) occurring in patients with FAP were intramucosal cancers or adenomas, except two cases that invaded deeper than the submucosal layer and one case of undifferentiated adenocarcinoma. Therefore, it is obvious that the prognoses of patients with FAP with gastric neoplasms were excellent. In addition, there remains disparity between Western and Japanese histopathologists in the conceptual approach to histopathologic evaluation of neoplastic lesions in the upper gastrointestinal tract because of a difference in the classification of gastric intramucosal neoplasms between JCGC and the World Health Organization classification [18,37]. Therefore, most of the gastric cancers in patients with FAP in this study were high-grade dysplasia except for 2 invasive gastric cancers in western countries. Hence, there may be discrepancies in the interpretation of the outcome of 
this study between Japan and western countries. Furthermore, the high proportion of atrophic gastritis in Japan may result in a higher incidence of gastric neoplasms than that in western countries. However, we believe that early detection and treatment for gastric neoplasms leads to better prognoses for patients with FAP. This study had a number of limitations, as follows: this was a retrospective study conducted in a single center, using a non-integrated follow-up method, and providing insufficient data of $H$. pylori infection.

\section{Conclusion}

In conclusion, FGP and atrophic gastritis in background gastric mucosa are significant risk factors for gastric neoplasms in patients with FAP. However, the prognoses of patients with FAP who have FGP and gastric neoplasms were satisfactory because of appropriate intervention using EGD. We recommend annual surveillance EGD for patients with FAP having FGP and gastric neoplasms and careful observation of these patients while taking into consideration the macroscopic characteristics of FGP, a condition that can develop into gastric neoplasms.

\section{Acknowledgements}

This study was supported by Grant-in-Aid for the Japan Agency for Medical Research and Development.

\section{Competing interests}

None

\section{References}

[1] Bussey HJR. Familial Polyposis Coli. Baltimore, Maryland: The Johns Hopkins University Press; 1975: 1-104

[2] Iwama T, Tamura K, Morita T et al. A clinical overview of familial adenomatous polyposis derived from the database of the Polyposis Registry of Japan. Int J Clin Oncol 2004; 9: 308-316

[3] Hauser G. Uber Polyposis intestinalis adenomatosa und deren beziehungen zur Krebsentwicklung. Arch F Kin Med 1895; 55: 429-448

[4] Utsunomiya J, Maki T, Iwama T et al. Gastric lesion of familial polyposis coli. Cancer 1974; 34: 745 - 754

[5] Watanabe H, Enjoji M, Yao T et al. Gastric lesions in familial adenomatosis coli: Their incidence and histologic analysis. Hum Pathol 1978; 9: 269-283

[6] Jagelman DG, DeCosse J], Bussey HJR. Upper gastrointestinal cancer in familial adenomatous polyposis. Lancet 1988; I: 1149-1151

[7] Sarre RG, Frost AG, Jagelman DG et al. Gastric and duodenal polyps in familial adenomatous polyposis: prospective study of the nature and prevalence of upper gastrointestinal polyps. Gut 1987; 28: 306-314

[8] Halstead JA, Harris E], Bartlett MK. Involvement of the stomach in familial polyposis of the gastrointestinal tract; report of a family. Gastroenterology 1950; 15: 763-770

[9] Domizo P, Talbot IC, Spigelman AD et al. Upper gastrointestinal pathology in familial adenomatous polyposis: results from a prospective study of 102 patients. J Clin Pathol 1990; 43: 738-743
[10] Hoffmann DC, Goligher JC. Polyposis of the stomach and small intestine in association with familia polyposis coli. Br J Surge 1971; 58 : $126-128$

[11] Spigelman AD, Williams CB, Talbot IC et al. Upper gastrointestinal cancer in patients with familial adenomatous polyposis. Lancet 1989; 2: $783-785$

[12] Sawada T, Muto T. Familial adenomatous polyposis: should patients undergo surveillance of the upper gastrointestinal tract? Endoscopy 1995; 27: 6-11

[13] Park SY, Ryu JK, Park JK et al. Prevalence of gastric and duodenal polyps and risk factors for duodenal neoplasm in Korean patients with familial adenomatous polyposis. Gut Liver 2011; 5: 46-51

[14] Murphy ES, Mireles M, Beltran A. Familial polyposis of the colon and gastric carcinoma. Concurrent conditions in a 16 -year-old boy. JAMA 1962; 179: $1026-1028$

[15] Iwama T, Mishima Y, Utsunomiys J. The impact of familial adenomatous polyposis on the tumorigenesis and mortality at the several organs; its rational treatment. Ann Surg 1993; 217: $101-108$

[16] Park JG, Park KJ, Ahn YO et al. Risk of gastric cancer among Korean familial adenomatous polyposis patients. Dis Colon Rectum 1992; 35: 996- 998

[17] Offerhaus G], Giardiello FM, Krush AJ et al. Risk of upper gastrointestinal cancer in familial adenomatous polyposis. Gastroenterology 1992; 102: $1980-1982$

[18] Japanese Gastric Cancer Association. Japanese classification of gastric carcinoma: 3rd English edition. Gastric Cancer 2011; 14: 101 - 112

[19] Kimura K, Takemoto T. An endoscopic recognition of the atrophic border and its significance in chronic gastritis. Endoscopy 1969; 1: $87-97$

[20] Japanese Gastric Cancer Association. Japanese gastric cancer treatment guidelines 2010 (ver. 3). Gastric Cancer 2011; 14: 113-123

[21] Gotoda T. Endoscopic resection of early gastric cancer. Gastric Cancer 2007; 10: $1-11$

[22] Oda I, Saito D, Tada M et al. A multicenter retrospective study of endoscopic resection for early gastric cancer. Gastric Cancer 2006; 9: $262-270$

[23] Gotoda T, Kondo H, Ono H et al. A new endoscopic mucosal resection procedure using an insulation-tipped electrosurgical knife for rectal flat lesions: report of two cases. Gastrointest Endosc 1999; 50: $560-$ 563

[24] Ono H, Kondo H, Gotoda T et al. Endoscopic mucosal resection for treatment of early gastric cancer. Gut 2001; 48: 225-229

[25] Shibata C, Ogawa H, Unno M et al. Clinical characteristics of gastric cancer in patients with familial adenomatous polyposis. Tohoku J. Exp. Med 2013; 229: 143-146

[26] Shimoyama S, Aoki F, Kaminishi M et al. Early gastric cancer development in a familial adenomatous polyposis patient. Dig. Dis. Sci 2004; 49: $260-265$

[27] Wu TT, Kornacki S, Rashid A et al. Dysplasia and dysregulation of proliferation in foveolar and surface epithelia of fundic gland polyps from patients with familial adenomatous polyposis. Am J Surg Pathol 1998; 22: $293-298$

[28] Abraham SC, Nobukawa B, Giardiello FM et al. Fundic gland polyps in familial adenomatous polyposis: Neoplasms with frequent somatic adenomatous polyposis coli gene alterations. Am J Pathol 2000; 157 : $747-754$

[29] Zwick A, Munir M, Ryan CK et al. Gastric adenocarcinoma and dysplasia in fundic gland polyps of a patient with attenuated adenomatous polyposis coli. Gastroenterology 1997; 113: 659-663

[30] Hofgartner WT, Thorp M, Ramus MW et al. Gastric adenocarcinoma associated with fundic gland polyps in a patient with attenuated familial adenomatous polyposis. Am J Gastroentrol 1999; 94: 2275 2281 
[31] Sekine S, Shimoda T, Hirohashi S et al. High-grade dysplasia associated with fundic gland polyposis in a familial adenomatous polyposis patient, with special reference to APC mutation profiles. Mod Pathol 2004; 17: $1421-1426$

[32] Toyooka M, Konishi M, Kikuchi-Yanoshita R et al. Somatic mutation of the adenomatous polyposis coli gene in gastroduodenal tumors from patients with familial adenomatous polyposis. Cancer Res 1995; 55: $3165-3170$

[33] Matsuda A, Matsuda T, Shibata A et al. Cancer incidence and incidence rates in Japan in 2008: a study of 25 population-based cancer registries for the Monitoring of Cancer Incidence in Japan (MCIJ) Project. Jpn J Clin Oncol 2013; 44: 388-396
[34] Yachida T, Nakajima T, Nonaka S et al. Characteristics and clinical outcomes of duodenal neoplasia in Japanese patients with familial adenomatous polyposis. J Clin Gastroenterol 2016; 50: DOI 10.1097/ MCG. 0000000000000555

[35] Ikeda Y, Saku M, Maehara Y et al. Effective follow-up for recurrence or a second primary cancer in patients with early gastric cancer. $\mathrm{Br}$. J. Surg 2005; 92: 235 -239

[36] Nozaki I, Nasu J, Kurita A et al. Risk factors for metachronous gastric cancer in the remnant stomach after early cancer surgery. World J. Surg 2010; 34: $1548-1554$

[37] Sekine $S$, Yoshida $H$, Jansen $M$ et al. The Japanese viewpoint on the histopathology of early gastric cancer. Adv Exp Med Biol 2016; 908: $331-346$ 